\title{
Thermal considerations in the cryogenic regime for the BNL double ridge higher order mode waveguide
}

\author{
Dhananjay K. Ravikumar, ${ }^{1,2,3, *}$ Yatming Than, ${ }^{1,3}$ Wencan Xu, ${ }^{1,3}$ and Jon Longtin ${ }^{2,3}$ \\ ${ }^{1}$ Collider-Accelerator Department, Brookhaven National Laboratory, Upton, New York 11973, USA \\ ${ }^{2}$ Department of Mechanical Engineering-Stony Brook University, New York 11790, USA \\ ${ }^{3}$ Center for Accelerator Science and Engineering-Stony Brook University, New York 11790, USA
}

(Received 18 April 2017; published 6 September 2017)

Brookhaven National Laboratory (BNL) has proposed to build an electron ion collider (EIC) as an upgrade to the existing Relativistic Heavy Ion Collider (RHIC). A part of the new design is to use superconducting radio frequency (SRF) cavities for acceleration, which sit in a bath of superfluid helium at a temperature of $2 \mathrm{~K}$. SRF cavities designed for the BNL EIC create a standing electromagnetic wave, oscillating at a fundamental frequency of $647 \mathrm{MHz}$. Interaction of the charged particle beam with the EM field in the cavity creates higher order modes (HOM) of oscillation which have adverse effects on the beam when allowed to propagate down the beam tube. HOM waveguides are thus designed to remove this excess energy which is then damped at room temperature. As a result, these waveguides provide a direct thermal link between room temperature and the superconducting cavities adding a static thermal load. The EM wave propagating through the warmer sections of the waveguide creates an additional dynamic thermal load. This study calculates these thermal loads, concluding that the dynamic load is small in comparison to the static load. Temperature distributions are mapped on the waveguide and the number of heat intercepts required to efficiently manage thermal loads have been determined. In addition, a thermal radiation study has been performed and it is found that this contribution is around three orders of magnitude smaller than the static conduction and dynamic loads.

DOI: 10.1103/PhysRevAccelBeams.20.093201

\section{INTRODUCTION}

The stringent demands of modern day high energy physics research calls for a powerful particle accelerator. Realizing this goal has seen a significant increase in the use of superconducting structures, bringing about drastic improvements in the performance of particle accelerators. Large cryogenic plants are built to meet the demands of these machines and efficient cryogenic management of thermal loads are of paramount importance, thus motivating thermal studies on structures that will load the cryogenic system.

The Relativistic Heavy Ion Collider has, for the past two decades helped search for answers to some of the most challenging problems in modern physics [1]. It is a unique facility, capable of delivering spin polarized proton beams, to explore the mechanism behind the proton's spin as well as delivering beams of gold ions, to shed light on the quark gluon plasma and the early universe. Counter rotating beams of charged particles are steered to collide head on, creating exotic states of matter. These states are very short

\footnotetext{
ravikumar@bnl.gov
}

Published by the American Physical Society under the terms of the Creative Commons Attribution 4.0 International license. Further distribution of this work must maintain attribution to the author(s) and the published article's title, journal citation, and DOI. lived and, undergo a series of decays before reaching the detector chambers that surround the interaction point. Data thus recorded by the detectors are analyzed and, their initial states reconstructed, to unravel the workings of the universe. The future of the RHIC program as seen by the nuclear physics community is to upgrade to an electron ion collider $[2,3]$ that will collide a beam of hadrons with a beam of electrons, rather than two hadron beams. The path of upgrading to an EIC at BNL (shown in Fig. 1 [4]) involves the design and construction of a linear accelerator for the electron beam [3], in addition to the existing hadron acceleration complex in the form of RHIC and its preaccelerators. To accelerate charged particle beams, the choice has been made based on [5] to use superconducting radio frequency (SRF) cavities, with a fundamental operating frequency of $647 \mathrm{MHz}$. Called the BNL4, the cavity works by setting up a standing EM wave [6,7], fed through a power coupler from an rf source. The incoming beam of charged particles interacts with the gradients in the EM field and is accelerated. For a beam travelling in orbit in an ideal, lossless cavity there are no instabilities. However in the real system, wakefields are produced that generate longitudinal components of the electric field. Wakefields with oscillating frequencies equal to integral multiples of the cavity's eigenfrequency are called higher order modes (HOM). HOMs adversely affect the beam's motion which results in energy loss. The propagation of HOMs down the beam tube must thus be arrested to minimize these effects. 


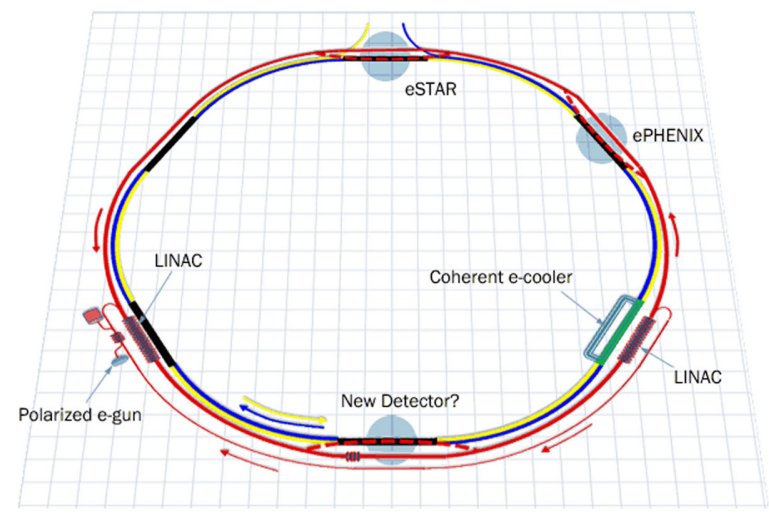

FIG. 1. Figure showing layout of the proposed electron ion collider at BNL.

One approach is to damp the HOM energy i.e., to convert it to heat which can then be removed by cooling the structure. This cannot be done in the cryogenic section, however, as the BNL4 cavities are designed to work in a bath of superfluid helium, operating at a temperature of $\approx 2 \mathrm{~K}$. The extra thermal load on the cryogenic system would be enormous: as a rule of thumb, $1 \mathrm{~W}$ of heat deposited at $2 \mathrm{~K}$ corresponds to $1 \mathrm{~kW}$ of primary AC power in the refrigerator [8]. The HOM energy per cavity is estimated to be around $20 \mathrm{~kW}$. Removing heat cryogenically would thus be very inefficient and costly. These challenges are overcome by designing waveguides with cutoff frequencies above the fundamental mode and below the HOM frequency. The waveguides act as a high-pass filter, allowing only the HOM modes to pass through providing a means to transport the HOM rf energy to room temperature where it can be damped and extracted as heat, at much lower expense. The analysis presented by $\mathrm{Xu}$ et al. in [9] summarizes the case for a double ridge profile for the HOM waveguide in Fig. 2. The cavity is housed in a helium vessel, which in turn, sits in a vacuum vessel called a cryomodule, which is being designed for the BNL4 cavity and waveguides. Pressures within the cryomodule are maintained at $10^{-7}$ Torr to minimize heat transfer via conduction and convection. Multilayer insulation is provided to act as a shield to thermal radiation. The presence of the waveguide however, which extends from the cold

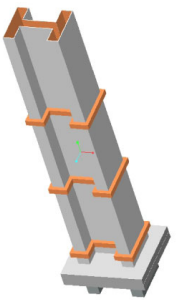

(a)

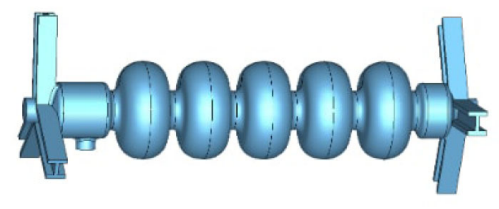

(b)
FIG. 2. Figure (a) shows the full length view of the waveguide. Figure (b) shows the waveguide atttached to the BNL4 cavity [10].
SRF cavity to room temperature offers an additional path for heat flow by linking the cold end with ambient conditions, which provides an additional load on the cryogenic cooling system. It is critical to assess the heat transfer through the waveguide for this reason which is the subject of this study.

The waveguide's design is depicted in Fig. 2. The length of the cryogenic section, i.e., from the cold end to the vacuum window, which is along the $x-$ axis is $60 \mathrm{~cm}$. The waveguide is made from stainless steel. To improve the electrical performance, the inner surfaces of the waveguide are copper coated with thicknesses in the $\mu \mathrm{m}$ range [11]. The cold section of the waveguide, extending $6 \mathrm{~cm}$ out of the SRF cavity is made of Niobium. The transition to the copper coated stainless steel $(\mathrm{Cu}-\mathrm{SS})$ section follows, extending for a length of another $54 \mathrm{~cm}$, outward to the vacuum window. The separate volumes are held in place by two flanges, one made of SS and the other made of Niobium.

\section{METHODOLOGY}

The total heat flow through the waveguide consists of two components. First is a static thermal load that develops because of the temperature difference between the two ends, i.e., $2 \mathrm{~K}$ and $300 \mathrm{~K}$. The static load conducted to the $2 \mathrm{~K}$ region is also affected by the thermal conductivity and thermal radiation. In addition, the fundamental mode extends into the waveguide and induces currents in the interior copper film. The electric surface resistance of copper increases with temperature, which, together with the rf wave results in Joule heating and presents a second dynamic load. One way to effectively manage the static and dynamic heat loads is to employ thermal intercepts [12-14] as shown in Fig. 2. The thermal intercepts are all located on the $\mathrm{Cu}-\mathrm{SS}$ section.

The static load is solved for first, yielding a temperature distribution along the waveguide that also can be used to perform optimization checks on the number, locations and operating temperatures of the intercepts. The dynamic load, which is a function of temperature is then introduced and both components are solved simultaneously resulting in maps of heat flow and temperature profiles on the waveguide. Since temperature gradients only start appearing below the vacuum window, the window itself and sections of the waveguide beyond have not been included to improve computational efficiency.

\section{A. Static load}

Temperature profiles and heat loads can be obtained as shown in [10] by solving the heat conduction equation:

$$
\frac{d T}{d t}=\frac{1}{\rho C_{p}} \nabla \cdot(k(T) \nabla T)
$$

separately for both the $\mathrm{Cu}$ and SS layers. Here, T is the temperature, $k(T)$ is the thermal conductivity as a function 


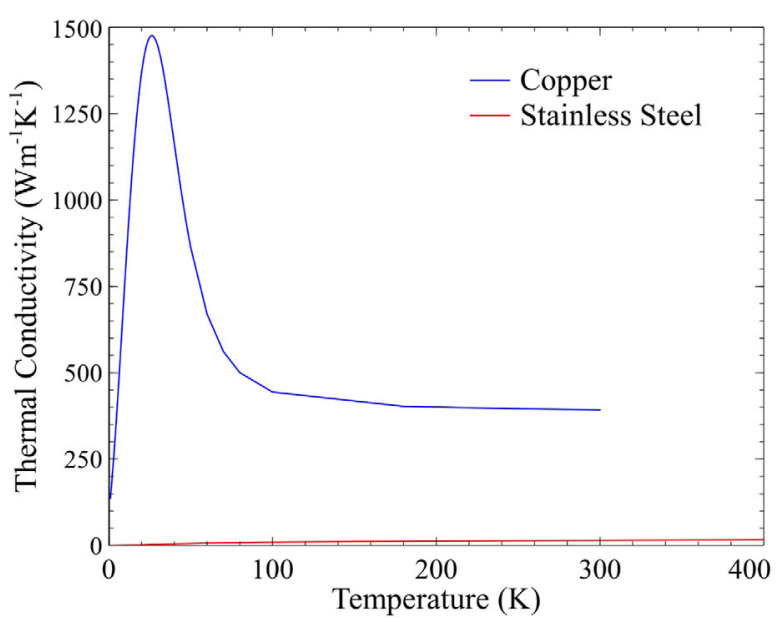

FIG. 3. Variation of thermal conductivities of copper and stainless steel with temperature.

of temperature, $\rho$ is the density and $C_{p}$ is the specific heat capacity. The presence of thermal intercepts makes this a 2-D problem since they generate temperature gradients along the $\mathrm{y}$ and $\mathrm{z}$ directions. The temperature dependence of thermal conductivity in $\mathrm{Cu}$ and SS must be considered as the thermal conductivity increases sharply, following a rapid fall-off with temperature (Fig. 3). These effects have been incorporated, using polynomial fits for the appropriate material in [15-17]. The locations of intercepts [shown in orange in Fig. 2(a)], their thickness and operating temperatures at those locations must thus be determined so that the intercepts act as a heat sink as opposed to an additional heat source. Intercepts are made of high purity oxygen free high conductivity (OFHC) copper, with a residual resistance ratio (RRR) of 500. The copper coating by comparison, has a RRR of 50 .

\section{B. Dynamic load}

The power dissipated by a traveling rf wave is calculated as described in [18] from:

$$
\begin{aligned}
P & =\int \frac{1}{2} H^{2} R_{s} d s, \\
R_{s} & =\frac{1}{\sigma \delta}
\end{aligned}
$$

where, $H$ is the magnetic field strength, $R_{s}$ is, in this application, the surface resistance of copper, $\sigma$ is the bulk resistivity, and $\delta$ is the skin depth. If $R_{s}$ in $\Omega$ and $H$ is in $\mathrm{A} / \mathrm{m}$, the integral in Eq. (2a) directly yields the power in watts.

The surface resistance of copper $\left(R_{s}\right)$, is a function of temperature and thus varies along the length of the waveguide (Fig. 4). The solution to Eq. (2a) is thus a function of both temperature and location and is used as an additional boundary condition to Eq. (1), which then captures the complete thermal loading. ANSYS is used to solve the

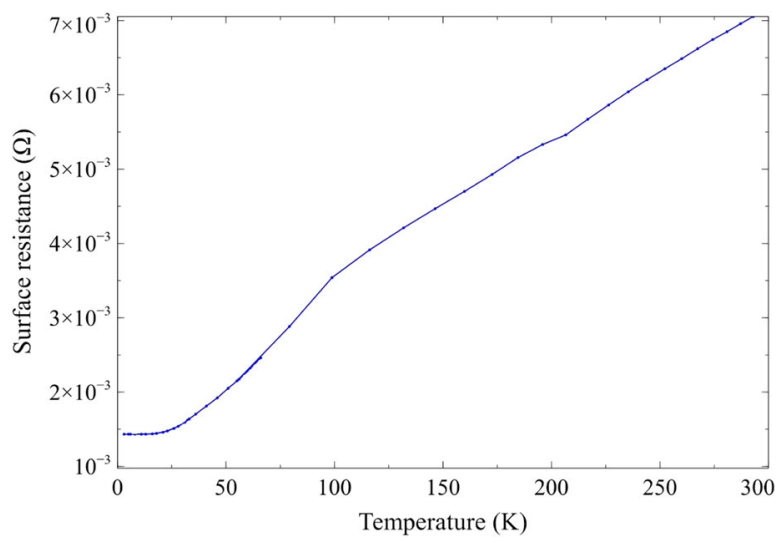

FIG. 4. Variation of surface resistance of copper with temperature.

resulting governing equations for the coupled ThermalEM problem. The absence of the high frequency structure simulator (HFSS) however, means that the Joule heating cannot be solved for directly by using ANSYS. Instead an alternative method was used to account for Joule heating, which was then fed back into ANSYS.

Though the waveguide has a cutoff frequency above that of the fundamental mode, some rf energy from the fundamental mode still extends through, decaying exponentially with distance. The HOM energy however, is transported through the waveguide, into a damper outside the cryomodule, where its energy is deposited. The major contribution towards the dynamic load thus comes from the decaying fundamental mode rather than the higher order modes. The ANSYS GUI has limited options to specify heat loads. So in order to replicate Joule heating, the coupled $\mathrm{SS}-\mathrm{Cu}$ conduction problem is solved and temperature distributions are obtained. Knowing values of temperature and the $\mathrm{H}$ field as a function of position, the dynamic load can be computed analytically using appropriate values of surface resistance in Eq. (2a). Heat flow as a function of temperature is thus evaluated and fed back into ANSYS by means of an Ansys Parametric Design Language (APDL) script.

\section{ANALYSIS AND RESULTS}

\section{A. Analytical model}

To ensure the computational model is self-consistent and to raise the level of confidence in the results obtained by the numerical techniques, analytical calculations of heat loads have been performed for a simplified version of the model. This also serves as a baseline test case for creating an efficient computational model. The heat conducted through the stainless and copper volumes can be calculated independently by reducing Eq. (1) for a steady state, 1D problem as:

$$
Q=\frac{A \int_{T_{2}}^{T_{1}} k(T) d T}{d x} .
$$




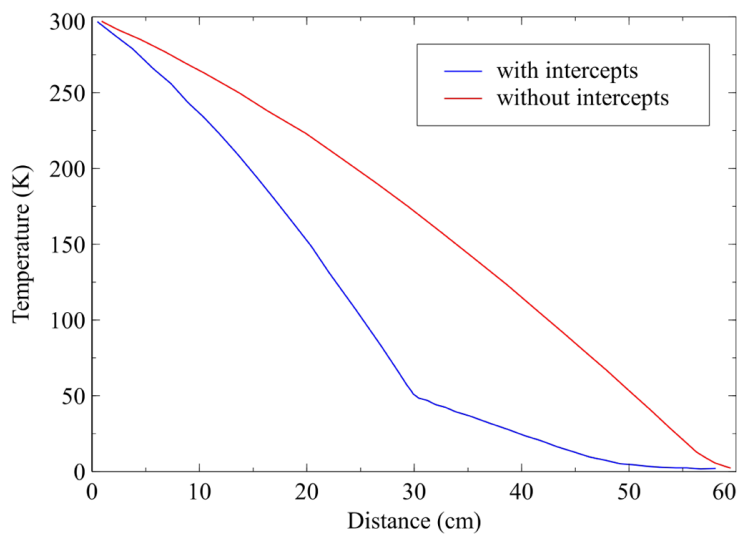

FIG. 5. Temperature as a function of position on the waveguide.

Here, $Q$ is the rate of heat flow in watts, $T_{1}$ and $T_{2}$ are fixed temperatures, which can either be the warm and cold ends or the temperatures of the intercepts in between, and $d x$ is the length or the distance between the two temperature limits of interest. The conduction area $A$ remains the same along the entire length. Determining the location of the intercepts are primarily constrained by temperature at those points. This is done to ensure that the intercept's operating temperature is actually lower than on the waveguide. To fix the operating conditions of the first intercept, a temperature distribution has to be obtained. The heat flux Q/A is constant, which allows us to make use of Eq. (3). Varying the length $d x$ with a sufficiently small interval gives us values for the conductivity integral as a function of position. Since the upper limit of the integral is known, the lower limit can now be found using numerical techniques, which yields the temperature as a function of position as shown in Fig. 5. In the case where intercepts are absent, it is observed that temperatures do not approach $6 \mathrm{~K}$ until the very end of the waveguide, which is not accessible to the intercepts since it is in the niobium region. Since the purpose of this study is to only compare temperature distributions, any temperature within the range for that of the intercepts would suffice. Bearing this in mind, two intercepts at $50 \mathrm{~K}$ and $6 \mathrm{~K}$ are positioned by using Fig. 5 as a guide in making sure that the operating temperatures are lower than the temperatures at corresponding locations.

To carry out the parametric study, the $6 \mathrm{~K}$ intercept was placed $5 \mathrm{~cm}$ above the stainless-steel flange and the location and temperature of the other, higher temperature (HT) intercept is varied to calculate heat loads, generating the plots in Fig. 6. Moving the HT intercept closer to the warm end would mean $d x_{\text {warm }}$ decreases and as the heat load, $Q$ varies inversely, the heat load increases. However, since the low temperature (LT) intercept is fixed, the distance between the HT intercept, $d x_{\text {cold }}$ increases, resulting in a lower heat load at the LT intercept. This behavior is clearly seen in Fig. 6. The variation of $Q$ at both the intercepts as a function of position for each fixed temperature of the HT intercept has been represented by lines of the same color. The two-intercept case has been considered

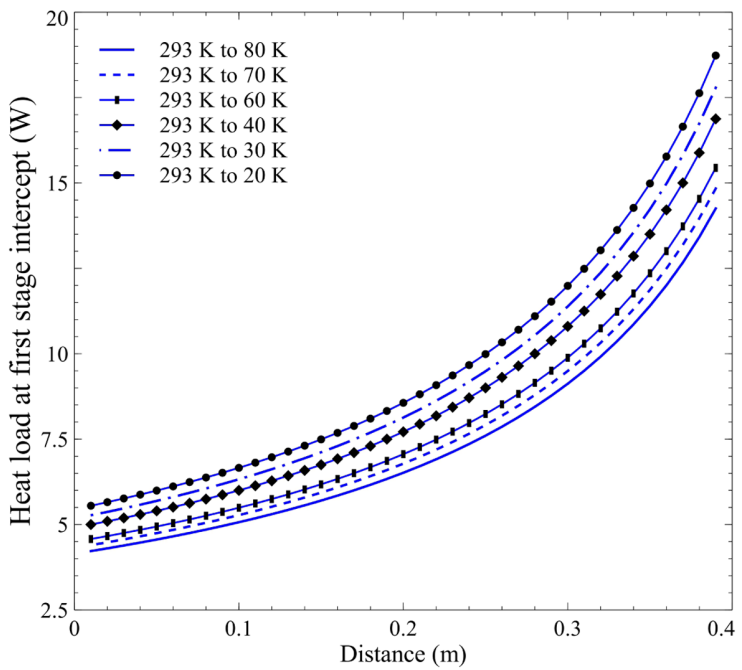

(a)

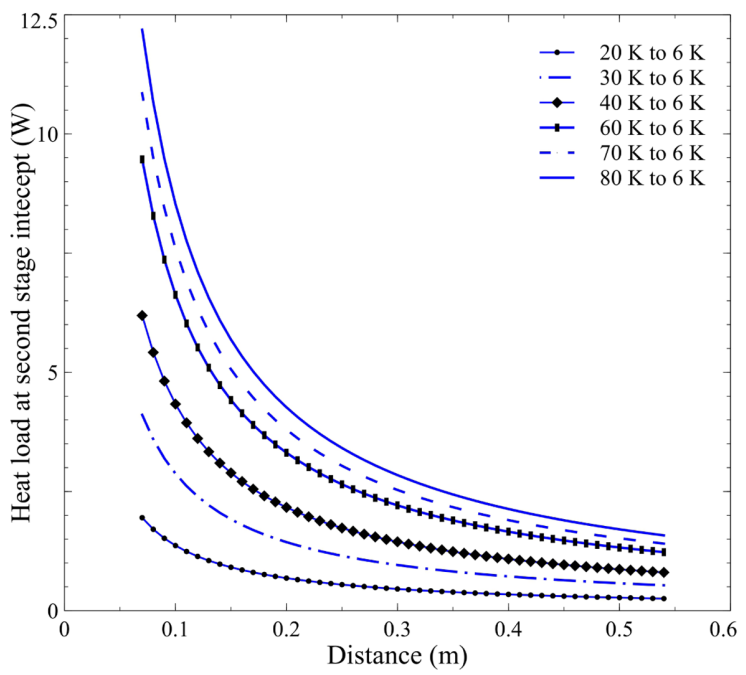

(b)

FIG. 6. Heat loads at first and second intercepts with change in distance and operating temperatures.

because of the relative ease of design and assembly of the thermal straps. Increasing the number of intercepts makes it easier to control the quantity of heat that can be intercepted between each temperature limit thereby enabling the design of a more efficient cryogenic system. However, there is an upper critical limit on the number of such intercepts that can be included in the design which results in a practical maximum of three thermal intercepts. Utilizing three intercepts, offers more flexibility in designing the cryogenic plant for example, at temperatures around $77 \mathrm{~K}$, using boiling nitrogen to remove heat from the HT intercept as opposed to using helium vapor is an option. Both the two and three intercept cases have been considered to aid in further R \& D efforts. The results of these studies are important parameters in optimizing the efficiency of the overall cryogenic system. The temperatures for the three station intercepts have been fixed, in addition to existing 


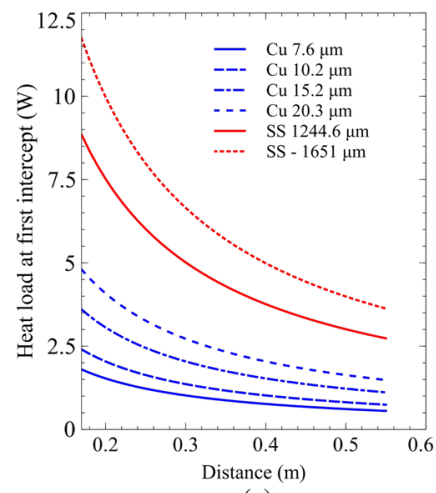

(a)

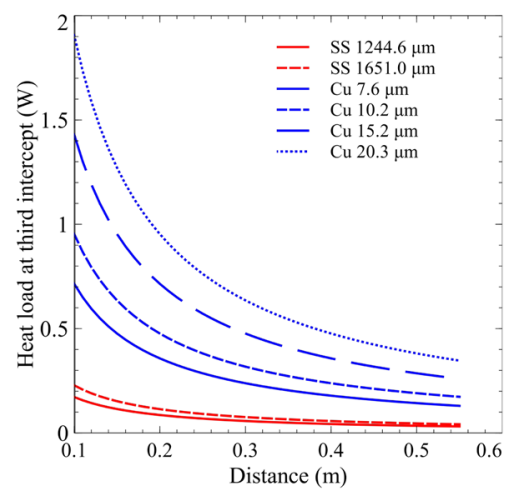

(c)

FIG. 7. Q variation with thickness of $\mathrm{Cu}$, SS and stage length (a) from $300 \mathrm{~K}$ to $85 \mathrm{~K}$ (b) from $85 \mathrm{~K}$ to $25 \mathrm{~K}$ and (c) from $25 \mathrm{~K}$ to $6.5 \mathrm{~K}$

constraints, keeping the larger picture of cryogenic system efficiency in mind. Fixing this now facilitates a parametric study of heat loads as a function of distance and a range of thickness of $\mathrm{SS}$ and $\mathrm{Cu}$, the findings of which are presented in Fig. 7. The heat conducted by the $\mathrm{SS}$ and $\mathrm{Cu}$ are comparable in Fig. 7(a) though the $\mathrm{Cu}$ is around three orders of magnitude thinner. As the temperature drops, the heat flow through the $\mathrm{Cu}$ starts to dominate because of the steep rise in thermal conductivity compared to that of SS. This is an important reason why the thickness of the $\mathrm{Cu}$ coating must be optimized. Being conservative from the thermal design point of view, a thickness of $20 \mu \mathrm{m}$ is set for the $\mathrm{Cu}$ since a setup that can manage heat from such a scenario will be valid for lower values of thickness, as supported by the findings in Fig. 7.

\section{B. Computational model}

It is possible to analytically compute heat loads but to map temperature distributions on the waveguide, Eq. (1) has to be solved in 2-D. Obtaining a proper temperature distribution is necessary before proceeding further with the analysis because the dynamic load is sensitive to temperature. Solving for temperature and heat fluxes while also incorporating thermal radiation for this geometry makes an analytical approach futile, favoring instead computational techniques.
TABLE I. Static heat load.

\begin{tabular}{lcc}
\hline \hline $\begin{array}{l}\text { Boundary } \\
\text { condition }\end{array}$ & $\begin{array}{c}\text { Temperature } \\
\text { (kelvin) }\end{array}$ & $\begin{array}{c}\text { Heat flow (Q) } \\
\text { (watts) }\end{array}$ \\
\hline Warm end & 300 & 12.17 \\
Intercept & 85 & -7.55 \\
Intercept & 25 & -3.15 \\
Intercept & 6.5 & -0.94 \\
Cold end & 2 & -0.53 \\
\hline \hline
\end{tabular}

Thermal simulations have been performed using ANSYS for both meshing and solution. The copper plating has been modeled as a shell. Intercept locations have been fixed based on the analysis in the previous sections and on other constraints such as ease of manufacturing and assembly in the cryomodule. The $85 \mathrm{~K}$ intercept is $20 \mathrm{~cm}$ from the warm end, the $25 \mathrm{~K}$ intercept is $15 \mathrm{~cm}$ further below and the $6.5 \mathrm{~K}$ intercept is $10 \mathrm{~cm}$ above the cold end.

To make sure results are independent of mesh size, a grid independence test [19] is carried out by varying the size of the mesh, usually by making it smaller by a factor of 2 and observing changes in the results. Once the sizes are in the region within which relative error in the results are minimized, it can be concluded that the results are independent of grid size. Taking all these effects into account, simulations for varying grid sizes were run and the results are summarized in Table I. The values of Q are obtained by computing reactions to each boundary condition. Heat flow into the waveguide $\left(Q_{i}\right)$ is positive and leaving the waveguide $\left(Q_{o}\right)$ is negative. A simple, yet effective way to check for self-consistency is to verify that Eq. (4) is satisfied

$$
\sum_{i=1}^{n} Q_{i}=\sum_{o=1}^{n} Q_{o}
$$

which is true for this case. The analytical study in the previous sections is validated by the fact that for the present combination of temperatures and distances, heat moves out of the system at the intercepts. Having determined the temperature distributions, shown in Fig. 8, this can be now used to facilitate computation of the dynamic load. Analytical computation is possible as described in Sec. II B. Prescribing Q(T) as a boundary condition has already taken into account the dependencies of the surface resistance on temperature and H field's decay. The trend in both these quantities are opposite: $R_{s}$ increases while " $\mathrm{H}$ " decreases. The changes in heat loads because of the additional boundary condition are reported in Table II. The dynamic load is asymmetric along the cross section but such small variations are neglected, justified by the fact that the dynamic load is only around $10 \%$ of the static load.

To complete the study, we estimate thermal radiation loads as well. Such a study is called for by the fact that the geometry is not optimized, i.e., it has a large cross section for a given perimeter, in addition to which there are 


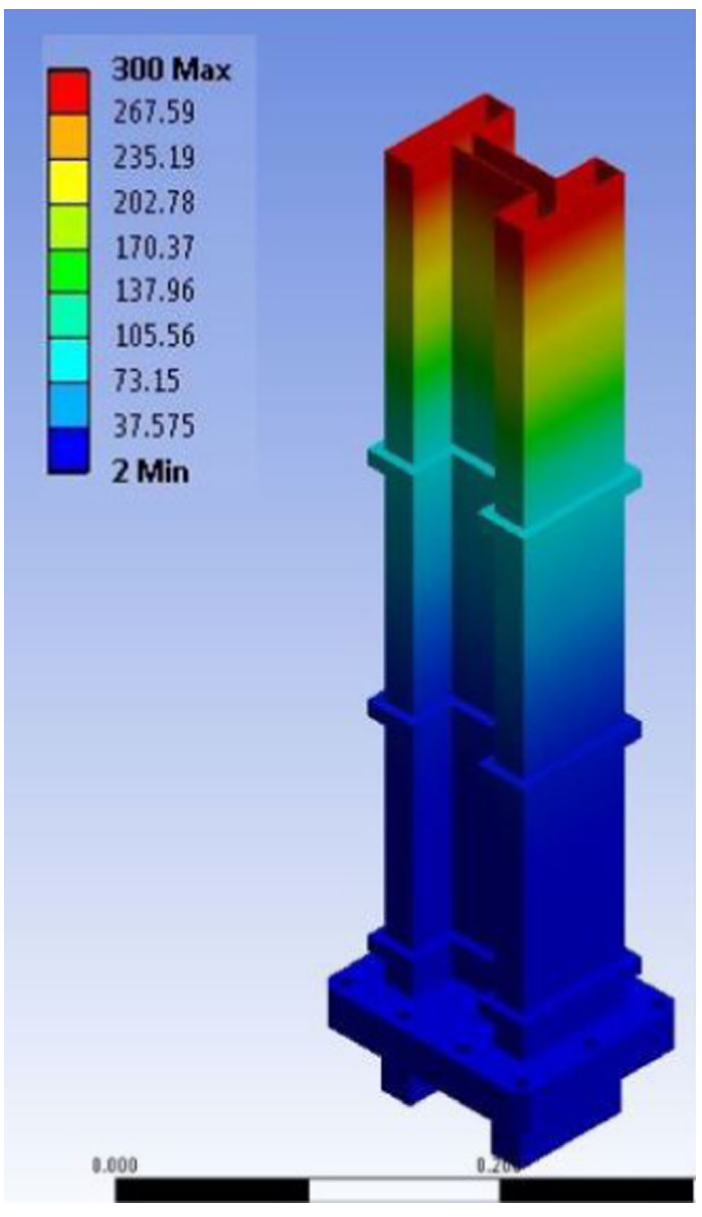

FIG. 8. Temperature distribution obtained numerically.

substantial changes in temperature. Such calculations become challenging owing to the complex nature of the geometry which makes view factors difficult to calculate. The radiation solver in ANSYS is used to perform the analysis which calculates view factors for each individual element to every other element as shown in [20] by:

$$
F_{i j}=\frac{1}{A_{i}} \iint \frac{\cos \theta_{i} \cos \theta_{j}}{\pi R^{2}} d A_{i} d A_{j}
$$

where, $F_{i j}$ is the portion of radiation leaving surface $i$ that falls on $j, \theta_{i}$, and $\theta_{j}$ are the polar angles formed by the line

TABLE II. Dynamic heat load.

\begin{tabular}{lcc}
\hline \hline $\begin{array}{l}\text { Boundary } \\
\text { condition }\end{array}$ & $\begin{array}{c}\text { Temperature } \\
\text { (kelvin) }\end{array}$ & $\begin{array}{c}\text { Heat flow (Q) } \\
\text { (watts) }\end{array}$ \\
\hline Warm end & 300 & 11.20 \\
Intercept & 85 & -8.29 \\
Intercept & 25 & -2.79 \\
Intercept & 6.5 & -0.66 \\
Cold end & 2 & -1.20 \\
Dynamic load & & 1.74 \\
\hline \hline
\end{tabular}

TABLE III. Radiation load.

\begin{tabular}{lcc}
\hline \hline Surface & Emissivity $(\epsilon)$ & Net radiation (watts) \\
\hline $\mathrm{SiC}$ & 0.9 & 0.44 \\
$\mathrm{Cu}$ plating & 0.05 & -0.38 \\
$\mathrm{Nb}$ waveguide & 0.9 & -0.01 \\
Cold Nb surface & 0.9 & -0.00357 \\
\hline \hline
\end{tabular}

$\mathrm{R}$ with surface normals, $n_{i}, n_{j}$, and $R$ is the line joining the two elemental areas $A_{i}$ and $A_{j}$.

The waveguide continues to extend outside of the cryomodule, where the $\mathrm{Cu}-\mathrm{SS}$ sections are replaced by silicon carbide ( $\mathrm{SiC})$, which acts as a damper, absorbing the HOM energy. For the radiation analysis, this has been modelled as a surface with very high emissivity, closing the upper end of the waveguide. Since all the radiation leaving the waveguide, into the cavity at the cold end must pass through the cross section, another surface closing the cold end is placed for ease in calculating net radiation load that will be distributed into the cavity. ANSYS is a radiosity solver, i.e., it solves for heat leaving the surfaces and balances them across all surface elements. So in this case, heat leaving the surface is positive and heat received by a surface is negative, as opposed to the convention for conduction. From Table III, it is observed that the heat received by the cold end is in the order of $10^{-3} \mathrm{~W}$, a negligible fraction of the total load. This can be attributed to the geometry of the waveguide. While the cross section is not optimized for the perimeter, there are two long ridges on either half.

\section{CONCLUSIONS}

A thermal study has been carried out for the double ridge HOM waveguide, designed as a part of the ongoing research and development for an electron-ion collider at Brookhaven National Laboratory. Static and Dynamic loads have been estimated and a solution for temperature and heat flux in the waveguide has been obtained numerically. The results are used to determine the number and optimal location of several heat sinks along the waveguide to improve the cooling and thus the performance of the device. Developing the analytical model will help obtain quick estimates of heat loads for future waveguide designs which can serve as check points for numerical models. Further work would involve modelling thermal straps from the heat stations to the final heat sink, using these results to perform optimization studies for the eRHIC cryogenic system and validating the numerical studies with experimental measurements.

\section{ACKNOWLEDGMENTS}

The authors would like to thank Yongfeng Gao, Chen $\mathrm{Xu}$, and Philipp Kolb for helpful discussions 
during the course of this work. The study has been supported by Brookhaven Science Associates, LLC under contract No. DE-SC0012704 with the U.S. Department of Energy.

[1] RHIC User community, The case for continuing RHIC operations, white paper.

[2] A. Accardi et al., Electron Ion Collider: The Next QCD Frontier - Understanding the glue that binds us all, arxiv: $1212.1701 \mathrm{v} 3$.

[3] E. C. Aschenauer et al., eRHIC Design Study: An ElectronIon Collider at BNL, arxiv:1409.1633.

[4] https://www.bnl.gov/rhic/eic.asp.

[5] W. Xu, I. Ben-Zvi, T. Roser, and V. Ptitsyn, Frequency choice of eRHIC SRF LINAC, Web document.

[6] P. Schmüser, Basic Principles of rf superconductivity and superconducting cavities, SRF 2003 (unpublished).

[7] J. Delayen, RF Superconductivity lectures at USPAS (unpublished).

[8] B. J. Holzer, Higher-order Modes and Heating, arxiv: $1501.07103 \mathrm{v} 1$.

[9] W. Xu et al., Ridge waveguide HOM damping scheme for high current SRF cavity, Proceedings of IPAC 2016,
Busan, Korea, http://accelconf.web.cern.ch/accelconf/ ipac2016/papers/wepmr042.pdf.

[10] D. Ravikumar, Y. Than, W. Xu, and J. Longtin, Heat transfer analysis in a higher order mode waveguide for the electron-ion collider at BNL, Proceedings of TFEC 2017, Nevada, USA (to be published).

[11] T. Treado, Power couplers for the ILC as noted in the original manuscript, Proceedings of the 22nd Particle Accelerator Conference, PAC-2007, Albuquerque, NM (IEEE, New York, 2007).

[12] P. Kittel, Advances in Cryogenic Engineering Parts A \& B (Springer, New York, 1998), p. 461.

[13] T. Hiatt et al., Proceedings of the 19th Particle Accelerator Conference, Chicago, IL, 2001 (IEEE, Piscataway, NJ, 2001).

[14] V. Parma, Cryostat Design, arxiv:1501.07154.

[15] C. Y. Ho and T. K. Chu, CINDAS Report No. 45.

[16] C. Y. Ho, R. W. Powell, and T. K. Chu, Thermal Conductivity of the Elements, J. Phys. Chem. Ref. Data 1, 279 (1972).

[17] NIST cryogenic database, from the website.

[18] D. Alesini, Power coupling, arxiv:1112.3201.

[19] Y. Jaluria, Computational Heat Transfer (CRC Press, New York, 2002), p. 321.

[20] F. P. Incropera and D. P. DeWitt, Fundamentals of Heat and Mass Transfer (Wiley India, New Delhi, 2007), p. 791. 